\title{
Editorial
}

\section{Management of rare diseases}

This Special Issue of Translational Science in Rare Diseases comprises seven articles on "Practical Management of Genetic Conditions". These articles represent a timely contribution to the field of clinical genetics.

Rare diseases are defined differently according to the specific jurisdiction responsible for their definition. In the United States, they are defined by the Food and Drug Administration as any condition that affects less than 200,000 persons in the country (for a prevalence of about 1 in 1,600). In Europe, the European Medicines Agency specifies a prevalence of less than 5 in 10,000 people (equivalent to less than 1 in 2,000), while in Japan the Ministry of Health, Labour and Welfare defines them as any condition affecting less then $<50,000$ individuals in the country (equivalent to less than 1 in 2,500). Although individually they are by definition rare, there are up to 8,000 of them, and they affect about $8 \%$ of the population, for a total of 25 million people in the United States alone. Rare diseases are thus collectively common.

The Orphan Drug Act of 1983 decreased barriers and provided incentives to develop and market new drugs to treat rare diseases. Although in the past about one-third of all FDAapproved drugs were orphan [1], over the last three years that number has ranged from 40-50\% (https://www.fda.gov/Drugs/DevelopmentApprovalProcess/DrugInnovation/default.htm, accessed October 25, 2018). However, only about $5 \%$ of rare diseases are treatable by FDA-approved drugs.

A study analyzing data collected up to 1983 attempted to measure the efficacy of treatment for Mendelian disorders by scoring the impact of each condition in seven different categories: lifespan, reproductive capability, somatic growth, intellectual development, learning ability, capacity to work, and cosmetic effect. For a group of 351 single gene disorders selected using every third entry from the 5th edition of McKusick's Mendelian Inheritance in Man [2], the lifespan was extended to normal in only $15 \%$ of conditions for which longevity was reduced [3]. An analysis in 1998 of 372 Mendelian disorders selected from the 7th edition of The Metabolic and Molecular Bases of Inherited Disease found that $12 \%$ of diseases (46/372) showed a complete response to treatment, 54\% (200/372) showed a partial response, while 39\% (126/372) showed no response [4].

Inborn errors of metabolism represent a model for the treatment of genetic disease [4].

A subgroup analysis of the aforementioned 1983 study included 65 inborn errors of metabolism, for which the pathogenesis of disease was better understood than for other Mendelian disorders. It found that $12 \%$ of inborn errors (8/65) were fully treatable, a partial response was seen in $40 \%(26 / 65)$, and no response in 48\% (31/65) [3]. The same group a decade later in 1993 performed a follow-up analysis, and found that the group of fully treatable conditions remained unchanged at $12 \%(8 / 64)$, while partially treatable conditions increased to 57\% (36/64), and untreatable conditions had decreased to $31 \%(20 / 64)$ [5]. A 25-year follow-up was published in 2008, although by now the original 65 inborn errors of metabolism had been reclassified into 57 distinct disease entities. By then, 22\% of inborn errors (12/57) had a full response to treatment, 54\% (32/57) showed a partial response, and 24\% (13/57) showed no response to therapy [6]. Therapeutic modalities included product replacement (31\%), substrate 
restriction (28\%), toxin removal (18\%), coenzyme supplementation (11\%), toxin inhibition (10\%), enzyme replacement therapy (10\%), liver transplantation (13\%), bone marrow transplantation (8\%), and kidney transplantation (3\%) [6].

In the early 1950s, phenylketonuria (PKU) became the first genetic condition to have specific treatment [7], and it became the model for the treatment of other inborn errors of metabolism. Although PKU does not predispose the patient to metabolic crises, other metabolic disorders such organic acidemias require different management approaches in the acute vs. chronic settings. Many lysosomal storage disorders have targeted therapy in the form of enzyme replacement therapy, substrate reduction therapy, pharmacological chaperones, or bone marrow transplantation. In this issue, experts in organic acidemias and lysosomal disorders, respectively, will describe how they approach management of these conditions from a practical standpoint. One added layer of difficulty in the management of these patients involves the transition of medical care from childhood to adulthood, especially considering that patients with metabolic disorders are living longer, both due to improved diagnostic as well as therapeutic strategies. An article is thus dedicated in this special issue to the subject of transitional care.

It is important to note that rare diseases can be difficult to diagnose and manage. This is not only because of their rarity, but also due to the lack of training, starting with very little exposure to them during medical school. This combination of findings contributes to the lack of trained specialists, either in developing countries, or in rural areas in developed countries. Thus, patients with rare diseases living in places with little access to medical care have difficulty not only in reaching a correct diagnosis, but also in being appropriately managed. The European Organisation for Rare Diseases (EURORDIS) conducted a series of surveys between 2002 and 2008; in particular, the EurordisCare 3 survey assessed access to care for 16 rare diseases in 23 different European countries. Of 20,000 questionnaires sent, responses were obtained for 5,995 patients [8]. Access to the eight most needed medical services was difficult (12\%), very difficult (3\%), or impossible (11\%) for a total of $26 \%$ of patients [9]. For $11 \%$ of respondents who found that access to adequate medical care was impossible, $69 \%$ referred that lack of referral was the greatest barrier to accessing services, followed by unavailability of services (52\%), then waiting times $(41 \%)$, distance (33\%), and personal cost $(28 \%)$, with at least two of those reasons for lack of access found on average [9]. Almost one out of five patients (18\%) with a rare disease were rejected by their healthcare practitioner, in $85 \%$ of cases due to disease complexity [8]. In a subsequent survey, $44 \%$ of respondents reported that access to care only got worse over a period of two years [10]. One of the articles in this special issue addresses ways to increase access to genetic services in underserved populations.

Another roadblock for rare diseases is that many patients never reach a diagnosis, or diagnosis is delayed for many years. A 2013 online survey found that the average time to diagnosis was 7.6 years in the US, and 5.6 years in the UK [11]. In a French survey involving 448 respondents, the diagnosis was reached in $1-2$ years in $21.7 \%$, in $3-5$ years in $12.5 \%$, in $6-10$ years in $9.2 \%$, and in $>10$ years in $11.7 \%$ of patients [12]. The EurordisCare2 survey assessed diagnostic delays for eight rare diseases, with 18,000 questionnaires sent, and response from 5,980 patients [8]. A third of patients reported deleterious consequences from a delayed diagnosis, $47 \%$ received at least one misdiagnosis, and $7 \%$ were treated inappropriately based on this misdiagnosis. In $18 \%$ of cases, the affected individual or family first entertained the possibility of a rare disease, while in $23 \%$ of cases they had to identify the appropriate diagnostic structure themselves. Finally, $25 \%$ of patients had to travel to another region to obtain the confirmatory diagnosis, while $2 \%$ had to travel to another country [8]. Thus, even in those cases were a diagnosis was finally reached, it was only so after a long odyssey. In the current era of next-generation sequencing and with the advent of new genomic approaches, patients now can potentially have access to novel techniques to aid in the discovery of the molecular basis of their underlying rare diseases. Various approaches have recently been established to try to end the 
diagnostic odyssey of rare disease patients. Sometimes, these approaches lead to the discovery of a single individual with a completely novel genetic condition. Social media has occasionally been useful in bringing these patients together, and similarly, multiple platforms used to exchange phenotypic and genotypic information between physicians are now available. One article in this issue deals with ways to approach undiagnosed patients, while another addresses ways to approach management of patients where $n=1$.

Finally, we will end this issue with an article with an approach to genetic counseling in a tertiary care center. In the aforementioned EurordisCare2 survey, the genetic nature of the newly diagnosed disease was not communicated to the patient or family in $25 \%$ of cases, and genetic counseling was only offered in $52 \%$ of cases. In addition, in only $42 \%$ of cases it was suggested that the genetic nature of the disease should be informed to other family members, while on the other hand communicating this information to relatives led to the diagnosis of additional family members in $15 \%$ of cases. Thus, there is clearly room for improvement, and genetic counseling unquestionably plays an important role in the management of rare disease patients.

Translational Science of Rare Diseases is pleased to publish these articles, which will be indefinitely available on the Journal's website at https://www.iospress.nl/journal/translational-science-of-rarediseases/.

\section{References}

[1] D. Institute of Medicine (US) Forum on Drug Discovery, The Food and Drug Administration's Orphan Drug Program, National Academies Press (US), 2009. https://www.ncbi.nlm.nih.gov/books/NBK50974/ (accessed October 25, 2018).

[2] T. Costa, C.R. Scriver, and B. Childs, The effect of Mendelian disease on human health: a measurement, Am J Med Genet 21 (1985), 231-242.

[3] A. Hayes, T. Costa, C.R. Scriver and B. Childs, The effect of Mendelian disease on human health. II: Response to treatment, Am J Med Genet 21 (1985), 243-255.

[4] C.R. Scriver and E.P. Treacy, Is there treatment for "genetic" disease? Mol Genet Metab 68 (1999), 93-102.

[5] E. Treacy, B. Childs and C.R. Scriver, Response to treatment in hereditary metabolic disease: 1993 survey and 10-year comparison, Am J Hum Genet 56 (1995), 359-367.

[6] P.M. Campeau, C.R. Scriver and J.J. Mitchell, A 25-year longitudinal analysis of treatment efficacy in inborn errors of metabolism, Mol Genet Metab 95 (2008), 11-16.

[7] H. Bickel, J. Gerrard and E.M. Hickmans, Influence of phenylalanine intake on phenylketonuria, Lancet 265 (1953), 812-813.

[8] EURORDIS, The Voice of 12,000 Patients. Experiences and Expectations of Rare Disease Patients on Diagnosis and Care in Europe, Eurordis, 2009. https://books.google.com/books?id=13g4hrVlBnoC.

[9] Y. Le Cam, Patients' Needs and Expectations Concerning Access to Health Services_-The Eurordiscare 3 study. (2007). https://www.eurordis.org/IMG/pdf/ECRD2007-LeCam.pdf (accessed November 2, 2018).

[10] Access to treatment: unequal care for European rare disease patients, 2017. http://download2.eurordis.org.s3. amazonaws.com/Access $\% 20$ to\%20treatment/access $\% 20$ to $\% 20$ treatment $\% 20$ EN.pdf.

[11] Shire, Rare disease impact report: insights from patients and the medical community., The Journal of Rare Disorders (2014), 1-34.

[12] T. Heuyer, S. Pavan and C. Vicard, The health and life path of rare disease patients: results of the 2015 French barometer, Patient Relat Outcome Meas 8 (2017), 97-110. 